\title{
Early and late transcriptional responses to the low concentration of salicylic acid in Arabidopsis thaliana L. root
}

\author{
E. Elgaeva ${ }^{1 *}$, E. Zemlyanskaya ${ }^{1,2}$, D. Novikova ${ }^{1,2}$, V. Mironova ${ }^{1,2}$ \\ ${ }^{1}$ Novosibirsk State University, Novosibirsk, Russia \\ ${ }^{2}$ Institute of Cytology and Genetics SB RAS, Novosibirsk, Russia \\ *e-mail:elgaeva.liza@yandex.ru
}

Key words: SA, RNA-Seq, development, bioinformatics, transcriptome, concentration-dependent response

Motivation and Aim: Salicylic acid (SA) is a stress hormone in plants, also it was shown that SA regulates plant growth and development [1]. In majority of studies, the influence of high SA concentrations $(>100 \mu \mathrm{M})$, mainly on the aboveground part, and after a prolonged exposure to SA ( $\sim 24$ hours $)$ has been studied. As a result, moleculargenetic mechanisms of low SA concentrations remain obscure, the same is true for the mechanisms of SA response in the root and early transcriptional response to SA. The goal of this study was to describe transcriptome response to low SA concentrations in early and late response in $A$. thaliana root.

Methods and Algorithms: We used RNA-Seq data, generated from roots of 3 dag seedlings of A. thaliana after SA-treatment $(20 \mu \mathrm{M}, 1 \mathrm{~h}$ and $6 \mathrm{~h})$ and sequenced on IlluminaHiSeq4000 (BGI Tech, Hong Kong). RNA-Seq data analysis included quality control (FASTQC), preprocessing of the reads (Trimmomatic), mapping (STAR) and differential expression analysis (DESeq2, EdgeR). Functional annotation of differentially expressed genes (DEGs) was made with agriGO (http://systemsbiology.cau.edu.cn/agriGOv2) and DAVID (https://david.ncifcrf.gov/).

Results: We detected 1873 and 3662 DEGs in early and late response in A. thaliana root respectively. Low SA concentrations induced three times more genes than inhibits in both early and late response. Intriguingly, among the DEGs we found many genes providing for SA signaling under its high dosage. Consequently, functional enrichment analysis of DEGs showed many GO terms associated with defense and immune system even in the samples exposed to low-level SA for $1 \mathrm{~h}$ only. After 6h-treatment was found a term «SA metabolism», that assumed activation of SA catabolism in late SA response. Conclusion: Here we identified molecular targets of low SA concentrations in early and late response to low SA concentrations. Interesting that low level SA activates many defence reactions (through oxylipin, cytochrome, flavonoids, etc.). Next question is to compare the transcriptome response to low and high SA concentrations. Acknowledgements: The work was supported by RFBR 18-04-01130.

\section{References}

1. Vlot A.C., Dempsey D.A., Klessig D.F. (2009) Salicylic Acid, a Multifaceted Hormone to Combat Disease. Annu. Rev. Phytopathol. 47(1):177-206. 